\title{
ENSEMBLE AVERAGES CALCULATED FOR TWO-DIMENSIONAL SMOLUCHOWSKI-DEBYE ROTATIONAL DIFFUSION IN DC ELECTRIC FIELD*
}

\author{
W. Alexiewicz, S. Kielich and L. Wolejko \\ Nonlinear Optics Department, Institute of Physics, Adam Mickiewicz University \\ Grunwaldzka 6, 60-780 Poznani, Poland \\ (Received December 20, 1993)
}

\begin{abstract}
Ensemble averages $\langle\cos n \vartheta(t)\rangle(n=1,2,3,4)$ are calculated for the two-dimensional Smoluchowski-Debye rotational diffusion model in the presence of a DC reorienting electric field. The time dependence of $\langle\cos n \vartheta(t)\rangle$ is plotted for the special cases of (i) dipolar nonpolarizable molecules $(p \neq 0)$, and (ii) nondipolar anisotropically polarizable molecules $(p=0)$ and rectangular reorienting pulses.
\end{abstract}

PACS numbers: $77.40 .+\mathrm{i}$

\section{Introduction}

The theory of rotational diffusion of molecules essentially hinges on the calculation of statistical a verages describing the time-dependence of the reorientation of molecules in a medium acted on by external fields. For molecules of appropriately high symmetry, their rotational diffusion in a liquid medium is described by ensemble averages of the form

$$
\left\langle\Phi_{n}(\vartheta, t)\right\rangle=\langle\cos n \vartheta(t)\rangle, \quad n=1,2, \ldots
$$

dependent only on the polar angle $\vartheta(t)$ subtended by the symmetry axis of the molecule and the direction of the external reorienting field. These averages play an important role in effects of dielectric dipolar relaxation $(n=1)$, in Kerr's effect, consisting in the induction of optical birefringence in naturally isotropic media under the action of an external electric field $(n=2,4)$ as well as in many phenomena of nonlinear molecular optics [1-4].

*This work was performed within the framework of project PB 201309101 of the Committee for Scientific Research. 
Recently, Coffey et al. [5-7], starting from the stochastic Langevin equation for two-dimensional rotational diffusion (the moleculc can reorient in one plane only!) have derived analytical expressions for the averages $\left\langle\Phi_{1}(\vartheta, t)\right\rangle,\left\langle\Phi_{2}(\vartheta, t)\right\rangle$ in the presence of DC as well as cosine electric fields. They also have proposed solutions of the more general Fokker-Planck-Kramers equation for an ensemble of noninteracting molecules at planar rotation, taking into account the moment of inertia of the molecule.

Their method has been applied by Déjardin and Debiais $[8,9]$ to the description of changes in the refractive index of a liquid, acted on by the superposition of a DC and an AC electric field. This permitted the plotting of Cole-Cole diagrams for the real and imaginary parts of the birefringence at the frequencies $\omega$ and $2 \omega$ and has corroborated the importance of inertial effects in the range of high frequency electric fields.

In the present work we describe a procedure giving the evolution in time of the nonlinear functions $(n>2)$ of two-dimensional rotation for arbitrary shapes of the reorienting electric pulses and quite general shapes of the external potential (Eq. (2)).

However, our procedure does not take inertial effects into account. It nonetheless provides a consistent description of experimental studies of nonlinear molecular optical effects performed with the use of laser pulse techniques.

We moreover present graphs of two-dimensional rotation under the action of rectangular reorienting pulses.

\section{Theory}

We consider a statistical ensemble of molecules of a liquid with permanent dipole moments $\mu$ and linear electric polarizability anisotropy $\Delta \alpha=\alpha_{33}-\alpha_{11}$. For the sake of simplicity, we shall neglect electrostatic interactions between the molecules.

Their rotational diffusion resulting from Brownian motion and the action of an external time-dependent reorienting torque $N(\vartheta, t)$ is described by a stochastic Langevin equation. In the particular case of Brownian rotation in one plane only, described in terms of the polar angle $\vartheta(l)$ (we then deal with planar rotators), the Langevin equation takes the form $[5-7]$ :

$$
I \ddot{\vartheta}+I \beta \dot{\vartheta}=N(\vartheta, l)+\lambda(\iota)
$$

with $\ddot{v}$ - the angular acceleration, $\dot{v}$ - the angular velocity, and $I$ - the moment of inertia of a molecule. By $I \beta \dot{v}=\zeta \dot{v}$ we denote the frictional torque acting on the molecule in the liquid. In addition to the external torque $N(\vartheta, t)$ the molecule experiences the action of "white noise" with $\lambda(l)$ being a random torque resulting from stochastic collisions with other molecules.

We write the change in potential energy $V(\vartheta, t)$ of the molecule due to the action of the external reorienting field in the form of the sum

$$
V(\vartheta, t)=-\sum_{m=1}^{M} V_{m}(t) \cos m \vartheta, \quad m=1,2, \ldots, M
$$


where $\vartheta(l)$ is the polar angle between the symmetry axis of the molecule and the field. The shape of the coefficients $V(t)$ depends on how we clioose to specify the reorienting field. The molccule is thus acted on by the external torque

$$
N(\vartheta, t)=-\frac{\partial V(\vartheta, \iota)}{\partial \vartheta}=\sum_{m=1}^{M} m V_{m}(l) \sin m \vartheta .
$$

Essentially, it is our aim to calculate the statistical averages of the reorientational functions

$$
\left\langle\Phi_{n}\right\rangle \equiv\left\langle\Phi_{n}(\vartheta, t)\right\rangle=\langle\cos n \vartheta(t)\rangle,
$$

which describe the role of two-dimensional rotational molecular diffusion in phenomena of molecular optics making use of Eq. (1) and the general expression (3) for the torque. Into Eq. (1) we insert Eq. (3) and the time-derivatives of $v$ :

$$
\dot{\vartheta}=-\frac{\dot{\Phi}_{n}}{n \sqrt{1-\Phi_{n}^{2}}}, \quad \ddot{\vartheta}=-\frac{\ddot{\Phi}_{n}+n^{2} \Phi_{n} \dot{\vartheta}^{2}}{n \sqrt{1-\Phi_{n}^{2}}} .
$$

On averaging both terms of (1) we obtain

$$
\begin{aligned}
& I\left\langle\ddot{\Phi}_{n}\right\rangle+I n^{2}\left\langle\Phi_{n} \dot{\vartheta}^{2}\right\rangle+I \beta\left\langle\dot{\Phi}_{n}\right\rangle \\
& =-\sum_{m=1}^{M} m\left\langle V_{m}(l) \sin m \vartheta \sin n \vartheta\right\rangle-\langle\lambda(l) \sin n \vartheta\rangle .
\end{aligned}
$$

It is customary, on grounds of the stochastic properties of white noise, to assume that

$$
\langle\lambda(t) \sin n v\rangle=0 .
$$

Equation (6) simplifies considerably on neglecting the influence of the moment of inertia on the dynamics of the molecule. This amounts to neglecting the second time-derivative

$$
\left\langle\ddot{\Phi}_{n}\right\rangle=0
$$

and to assuming the angular velocity $\dot{v}$ and the average $\left\langle\Phi_{n}(t)\right\rangle$ as mutually independent statistically

$$
I\left\langle\dot{v}^{2} \Phi_{n}\right\rangle=I\left\langle\dot{v}^{2}\right\rangle\left\langle\Phi_{n}\right\rangle=k T\left\langle\Phi_{n}\right\rangle,
$$

where $k$ is Boltzmann's constant, and $T$ is the absolute temperature of the liquid. The assumptions (8), (9) mean that we consider times $t \gg I / \zeta$ sufficiently long for the molecules to have attained the steady state given by the Maxwellian velocity distribution.

With the assumptions (8), (9) inserted in (6) and after some simple trigonometry, the following set of differential-difference equations is obtained for the nonlinear and noninertial response of the ensemble of dipolar, polarizable and noninteracting molecules:

$$
\left\langle\dot{\Phi}_{n}\right\rangle+\frac{n^{2} k T}{I \beta}\left\langle\Phi_{n}\right\rangle-\sum_{m=1}^{M T} \frac{m V_{m}(l)}{I \beta}\left(\left\langle\Phi_{m-n}\right\rangle-\left\langle\Phi_{m+n}\right\rangle\right)=0 .
$$


One notes that the averages $\left\langle\Phi_{n}\right\rangle$ are recurrentially related by linear differential equations, involving rotational relaxation times of the two-dimensional rotator $\tau_{n}$ :

$$
\tau_{n}=\frac{I \beta}{n^{2} k T}, \quad n=1,2, \ldots
$$

The relaxation times $\tau_{n}$ should not be confounded with the woll known relaxation timcs $^{(3 \mathrm{D})} \tau_{n}$ of three-dimensional rotational diffusion $[1,4]$ :

$$
{ }^{(3 \mathrm{D})} \tau_{n}=\frac{I \beta}{n(n+1) k T}=\frac{{ }^{(3 \mathrm{D})} \tau_{1}}{n(n+1)} \text {. }
$$

The formal solution of the set of equations (10) on imposition of the initial condition $\left\langle\Phi_{n}(t=0)\right\rangle=\left\langle\Phi_{n}\right\rangle_{0}$ takes the form

$$
\begin{aligned}
& \left\langle\Phi_{n}(t)\right\rangle=\left\langle\Phi_{n}\right\rangle_{0} \mathrm{e}^{-t / \tau_{n}} \\
& \quad+\frac{n}{2 \tau_{1}} \sum_{m=1}^{M} \int_{0}^{t} q_{m}(u) \mathrm{e}^{-(t-u) / \tau_{n}}\left(\left\langle\Phi_{m-n}(u)\right\rangle-\left\langle\Phi_{m+n}(u)\right\rangle\right) \mathrm{d} u
\end{aligned}
$$

where we have introduced the dimensionless $m$-th reorientational parameter of the molecule

$$
q_{m}(t)=\frac{m V_{m}(t)}{k T} .
$$

The solution $(13)$ is useful if $q_{m}(l) \ll 1$, a condition very well fulfilled in experiments on molecular optics when the averages $\left\langle\Phi_{n}(t)\right\rangle$ are easily obtainable for arbitrary $n$ and a given potential (2), with arbitrary accuracy, by the Piccard method of successive approximations. The formula (13) tells us how the time evolution of the external potential $V(t)$ affects the time evolution of the averages $\left\langle\Phi_{n}(l)\right\rangle$. In what follows we shall perform calculations of the $\left\langle\Phi_{n}(l)\right\rangle$ for molecules in a DC electric reorienting ficld.

The calculation of the avcrages $\left\langle\Phi_{n}(l)\right\rangle$ from Eqs. (6) with the assumptions (8), (9) is equivalent to the search for the orientation probability function of two-dimensional rotation $f(\vartheta, t)$ in the form of an expansion

$$
f(\vartheta, t)=\sum_{n=0}^{\infty} a_{n}(t) \mathrm{e}^{-\mathrm{i} n \vartheta}
$$

having recourse to the Smoluchowski equation of two-dimensional rotational diffusion

$$
\frac{\partial f(\vartheta, t)}{\partial t}=\frac{k T}{\zeta} \frac{\partial}{\partial \vartheta}\left[\frac{\partial f(\vartheta, t)}{\partial \vartheta}-\frac{N(\vartheta, t)}{k T} f(\vartheta, t)\right] .
$$

Equation (16) was first used by Debye in his classical theory of dielectric dipolar relaxation $[1,10]$. 


\section{The relaxation functions of two-dimensional rotational diffusion of molecules in an electric ficld}

\subsection{The general case} is simply

If the molecule is in a reorienting electric field $E(t)=E g(t)$, the torque (3)

$$
N(\vartheta, t)=-\mu E g(l) \sin \vartheta-\Delta \alpha E^{2} g(t)^{2} \sin 2 \vartheta-\ldots
$$

For the torque (17), the counterpart of Eqs. (10) takes the form

$$
\begin{aligned}
& \left\langle\dot{\Phi}_{n}\right\rangle+\frac{1}{\tau_{n}}\left\langle\Phi_{n}\right\rangle+\frac{n p}{2 \tau_{1}} g(l)\left[\left\langle\Phi_{n+1}\right\rangle-\left\langle\Phi_{n-1}\right\rangle\right] \\
& +\frac{n q}{2 \tau_{1}} g^{2}(t)\left[\left\langle\Phi_{n+2}\right\rangle-\left\langle\Phi_{n-2}\right\rangle\right]=0
\end{aligned}
$$

where we have introduced the classical Langevin-Benoit parameters of molecular reoricntation [11]:

$$
p \equiv \frac{\mu E}{k T}, \quad q \equiv \frac{\Delta \alpha E^{2}}{2 k T} .
$$

A formal solution of the set of Eqs (18) has the form

$$
\begin{aligned}
& \left\langle\Phi_{n}(t)\right\rangle=\left\langle\Phi_{n}\right\rangle_{0} \mathrm{e}^{-t / \tau_{n}}+\frac{n}{2 \tau_{1}} \int_{0}^{t} \mathrm{e}^{-(t-u) / \tau_{n}}\left\{p g(u)\left[\left\langle\Phi_{n-1}(u)\right\rangle-\left\langle\Phi_{n+1}(u)\right\rangle\right]\right. \\
& \left.\quad+q g^{2}(u)\left[\left\langle\Phi_{n-2}(u)\right\rangle-\left\langle\Phi_{n+2}(u)\right\rangle\right]\right\} \mathrm{d} u .
\end{aligned}
$$

On neglecting the anisotropy of polarizability of the molecules $(q=0)$ in (18) and on assuming the unit step function $g(l)$ for the electric pulse, the above equations become identical with the equation (B7) of Coffcy's paper [5], whereas for $q \neq 0$ and the step function they bccome equivalent to the equations (9.21) of the paper by Watanabe and Morita [2].

It is our aim to calculate the averages $\left\langle\Phi_{n}(l)\right\rangle$ with an accuracy to the fourth power of the field strength $E^{4}$ of the pulse on the assumption of $p \gg 1, q \gg 1$. From (18) we get the set

$$
\begin{aligned}
& \left\langle\dot{\Phi}_{1}\right\rangle+\frac{1}{\tau_{1}}\left[1-\frac{1}{2} q g^{2}(l)\right]\left\langle\Phi_{1}\right\rangle+\frac{p g(t)}{2 \tau_{1}}\left(\left\langle\Phi_{2}\right\rangle-1\right)+\frac{q g^{2}(t)}{\tau_{1}}\left\langle\Phi_{3}\right\rangle=0, \\
& \left\langle\dot{\Phi}_{2}\right\rangle+\frac{1}{\tau_{2}}\left\langle\Phi_{2}\right\rangle+\frac{p g(t)}{\tau_{1}}\left(\left\langle\Phi_{3}\right\rangle-\left\langle\Phi_{1}\right\rangle\right)+\frac{q g^{2}(l)}{\tau_{1}}\left(\left\langle\Phi_{4}\right\rangle-1\right)=0 \\
& \left\langle\dot{\Phi}_{3}\right\rangle+\frac{1}{\tau_{3}}\left\langle\Phi_{3}\right\rangle+\frac{3 p g(t)}{\tau_{1}}\left(\left\langle\Phi_{4}\right\rangle-\left\langle\Phi_{2}\right\rangle\right)+\frac{3 q g^{2}(t)}{\tau_{1}}\left(\left\langle\Phi_{5}\right\rangle-\left\langle\Phi_{1}\right\rangle\right)=0 \\
& \left\langle\dot{\Phi}_{4}\right\rangle+\frac{1}{\tau_{4}}\left\langle\Phi_{4}\right\rangle+\frac{2 p g(l)}{\tau_{1}}\left(\left\langle\Phi_{5}\right\rangle-\left\langle\Phi_{3}\right\rangle\right)+\frac{2 q p^{2}(l)}{\tau_{1}}\left(\left\langle\Phi_{6}\right\rangle-\left\langle\Phi_{2}\right\rangle\right)=0
\end{aligned}
$$

where we have used the relations $\left\langle\Phi_{0}\right\rangle=1,\left\langle\Phi_{-1}\right\rangle=\left\langle\Phi_{1}\right\rangle$.

We now proceed to consider two particular cases, namely: dipolar but nonpolarizable molecules $(\mu \neq 0, \Delta \alpha=0)$; and nondipolar but anisotropically polarizable ones $(\mu=0, \Delta \alpha \neq 0)$. 
3.2. A parlicular case: dipolar $(p \neq 0)$ nonpolarizable $(q=0)$ molecules Equations (21) now take the form

$$
\begin{aligned}
& \left\langle\dot{\Phi}_{1}\right\rangle+\frac{1}{\tau_{1}}\left\langle\Phi_{1}\right\rangle+\frac{p g(l)}{2 \tau_{1}}\left(\left\langle\Phi_{2}\right\rangle-1\right)=0, \\
& \left\langle\dot{\Phi}_{2}\right\rangle+\frac{1}{\tau_{2}}\left\langle\Phi_{2}\right\rangle+\frac{p g(l)}{\tau_{1}}\left(\left\langle\Phi_{3}\right\rangle-\left\langle\Phi_{1}\right\rangle\right)=0, \\
& \left\langle\dot{\Phi}_{3}\right\rangle+\frac{1}{\tau_{3}}\left\langle\Phi_{3}\right\rangle+\frac{3 p g(l)}{\tau_{1}}\left(\left\langle\Phi_{4}\right\rangle-\left\langle\Phi_{2}\right\rangle\right)=0, \\
& \left\langle\dot{\Phi}_{4}\right\rangle+\frac{1}{\tau_{4}}\left\langle\Phi_{4}\right\rangle+\frac{2 p g(l)}{\tau_{1}}\left(\left\langle\Phi_{5}\right\rangle-\left\langle\Phi_{3}\right\rangle\right)=0 .
\end{aligned}
$$

If no field was acting at the moment of time $l=0$, that is if $g(0)=0$, the initial condition for the set (22) reads

$$
\left\langle\Phi_{0}(0)\right\rangle=1, \quad\left\langle\Phi_{n}(0)\right\rangle=0,
$$

and the solution of (22) is, with an accuracy to terms $\mathrm{O}\left(p^{4}\right)$, of the form

$$
\begin{aligned}
& \left\langle\Phi_{1}(t)\right\rangle=\frac{p}{2 \tau_{1}} \int_{0}^{t} \mathrm{e}^{-(t-u) / \tau_{1}} g(u) \mathrm{d} u \\
& \quad-\frac{p^{3}}{4 \tau_{1}^{3}} \int_{0}^{t} \mathrm{e}^{-(t-u) / \tau_{1}} g(u) \int_{0}^{u} \mathrm{e}^{-\left(u-u_{1}\right) / \tau_{1}} g\left(u_{1}\right) \\
& \quad \times \int_{0}^{u_{1}} \mathrm{e}^{-\left(u_{1}-u_{2}\right) / \tau_{1}} g\left(u_{2}\right) \mathrm{d} u_{2} \mathrm{~d} u_{1} \mathrm{~d} u \\
& \left\langle\Phi_{2}(t)\right\rangle=\frac{p^{2}}{2 \tau_{1}^{2}} \int_{0}^{t} \mathrm{e}^{-(t-u) / \tau_{2}} g(u) \int_{0}^{u} \mathrm{e}^{-\left(u-u_{1}\right) / \tau_{1}} g\left(u_{1}\right) \mathrm{d} u_{1} \mathrm{~d} u \\
& \quad-\frac{p^{4}}{4 \tau_{1}^{4}} \int_{0}^{t} \mathrm{e}^{-(t-u) / \tau_{2}} g(u) \int_{0}^{u} \mathrm{e}^{-\left(u-u_{1}\right) / \tau_{1}} g\left(u_{1}\right) \\
& \times \int_{0}^{u_{1}} \mathrm{e}^{-\left(u_{1}-u_{2}\right) / \tau_{2}} g\left(u_{2}\right) \int_{0}^{u_{2}} \mathrm{e}^{-\left(u_{2}-u_{3}\right) / \tau_{1}} g\left(u_{3}\right) \mathrm{d} u_{3} \mathrm{~d} u_{2} \mathrm{~d} u_{1} \mathrm{~d} u \\
& \quad-\frac{p^{4}}{4 \tau_{1}^{4}} \int_{0}^{t} \mathrm{e}^{-(t-u) / \tau_{2}} g(u) \int_{0}^{u} \mathrm{e}^{-\left(u-u_{1}\right) / \tau_{3}} g\left(u_{1}\right) \\
& \times \int_{0}^{u_{1}} \mathrm{e}^{-\left(u_{1}-u_{2}\right) / \tau_{2}} g\left(u_{2}\right) \int_{0}^{u_{2}} \mathrm{e}^{-\left(u_{2}-u_{3}\right) / \tau_{1}} g\left(u_{3}\right) \mathrm{d} u_{3} \mathrm{~d} u_{2} \mathrm{~d} u_{1} \mathrm{~d} u \\
& \quad \times \int_{0}^{u_{1}} \mathrm{e}^{-\left(u_{1}-u_{2}\right) / \tau_{2}} g\left(u_{2}\right) \int_{0}^{u} \mathrm{e}^{-\left(u_{2}-u_{3}\right) / \tau_{1}} g\left(u_{3}\right) \mathrm{d} u_{3} \mathrm{~d} u_{2} \mathrm{~d} u_{1} \mathrm{~d} u . \\
& \left\langle\Phi_{3}(t)\right\rangle=\frac{3 p^{3}}{4 \tau_{1}^{3}} \int_{0}^{t} \mathrm{e}^{-(t-u) / \tau_{3}} g(u) \int_{0}^{u} \mathrm{e}^{-\left(u-u_{1}\right) / \tau_{2}} g\left(u_{1}\right) \\
& \times \int_{0}^{u_{1}} \mathrm{e}^{-\left(u_{1}-u_{2}\right) / \tau_{1}} g\left(u_{2}\right) \mathrm{d} u_{2} \mathrm{~d} u_{1} \mathrm{~d} u, \\
&
\end{aligned}
$$


The simplest case of an electric pulse is that of the DC electric reorienting field, switched on jumpwise at $t=0$ :

$$
g(t)=u(t)= \begin{cases}0 & \text { for } t<0 \\ 1 & \text { for } t>0\end{cases}
$$

where $u(l)$ is the unit step or IIeaviside function.

With (24), the two-dimensional rotation relaxational functions are obtained in the form

$$
\begin{aligned}
& \left\langle\Phi_{0}(t)\right\rangle=1, \\
& \left\langle\Phi_{1}(t)\right\rangle=\frac{1}{2} p\left(1-\mathrm{e}^{-t / \tau_{1}}\right)-\frac{1}{4} p^{3}\left(\frac{1}{4}-\frac{4}{9} \mathrm{e}^{-t / \tau_{1}}\right. \\
& \left.+\frac{7}{36} \mathrm{e}^{-t / \tau_{2}}+\frac{t}{3 \tau_{1}} \mathrm{e}^{-t / \tau_{1}}\right)+\mathrm{O}\left(p^{5}\right) \\
& \left\langle\Phi_{2}(t)\right\rangle=\frac{1}{8} p^{2}\left(1-\frac{4}{3} \mathrm{e}^{-t / \tau_{1}}+\frac{1}{3} \mathrm{e}^{-t / \tau_{2}}\right) \\
& -\frac{1}{8} p^{4}\left[\frac{1}{12}+\frac{1}{6} \mathrm{e}^{-t / \tau_{1}}-\frac{2981}{2700} \mathrm{e}^{-t / \tau_{2}}+\frac{17}{150} \mathrm{e}^{-t / \tau_{3}}+\mathrm{e}^{-3 t / \tau_{1}}\right. \\
& \left.-\frac{7}{27} \mathrm{e}^{-7 t / \tau_{1}}-\frac{73}{180} \frac{t}{\tau_{2}} \mathrm{e}^{-t / \tau_{2}}+\frac{1}{24}\left(\frac{t}{\tau_{2}}\right)^{2} \mathrm{e}^{-t / \tau_{2}}\right]+\mathrm{O}\left(p^{6}\right), \\
& \left\langle\Phi_{3}(t)\right\rangle=\frac{1}{4} p^{3}\left(\frac{1}{12}+\frac{1}{2} \mathrm{e}^{-t / \tau_{1}}+\frac{1}{15} \mathrm{e}^{-t / \tau_{3}}-\frac{13}{20} \mathrm{e}^{-t / \tau_{2}}\right)+\mathrm{O}\left(p^{5}\right), \\
& \left\langle\Phi_{4}(t)\right\rangle=\frac{1}{2} p^{4}\left(\frac{1}{192}-\frac{1}{2} \mathrm{e}^{-t / \tau_{1}}-\frac{13}{80} \mathrm{e}^{-t / \tau_{2}}\right. \\
& \left.+\frac{1}{135} \mathrm{e}^{-t / \tau_{3}}+\frac{1123}{1728} \mathrm{e}^{-t / \tau_{4}}\right)+\mathrm{O}\left(p^{6}\right) .
\end{aligned}
$$

When the rectangular pulse is switched on at $t=0$, the functions $\left\langle\Phi_{n}(t)\right\rangle$ (for $n=1,2,3,4)$ grow from their initial state value $\left\langle\Phi_{n}(0)\right\rangle$ to their steady state values $\left\langle\Phi_{n}(\mathrm{~s} . \mathrm{s})\right\rangle$ (for $t \gg \tau_{1}$ ), respectively

$$
\begin{aligned}
& \left\langle\Phi_{1}(\text { s.s. })\right\rangle=\frac{1}{2} p\left(1-\frac{1}{2} p^{2}\right)+\mathrm{O}\left(p^{5}\right) \\
& \left\langle\Phi_{2}(\text { s.s. })\right\rangle=\frac{1}{8} p^{2}\left(1-\frac{1}{12} p^{2}\right)+\mathrm{O}\left(p^{6}\right) \\
& \left\langle\Phi_{3}(\text { s.s. })\right\rangle=\frac{1}{48} p^{3}+\mathrm{O}\left(p^{5}\right) \\
& \left\langle\Phi_{4}(\text { s.s. })\right\rangle=\frac{1}{384} p^{4}+\mathrm{O}\left(p^{6}\right) .
\end{aligned}
$$

The rise in time of the functions (20) is plotted in Fig. 1.

We see that the time dependence of the ensemble avcrages $\left\langle\Phi_{n}(t)\right\rangle$ differ for different $n$. The functions $\left\langle\Phi_{1}(t)\right\rangle$ describing the linear rotational relaxation increase, and are always positive; they are dominated by the exponential growth of $1-\exp \left(t / \tau_{1}\right)$. A similar result has been reported recently by Cisto and Dudek [13]. The functions $\left\langle\Phi_{2}(t)\right\rangle$ undergo a change in sign for short times and then grow 

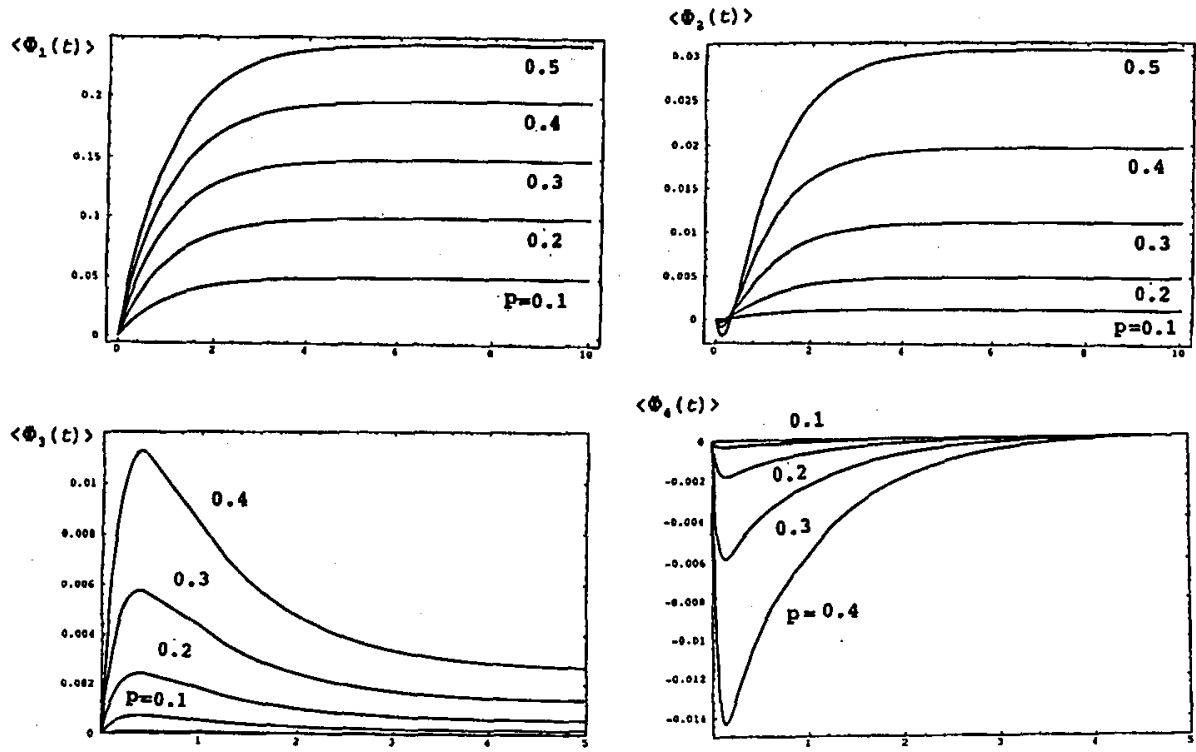

Fig. 1. Time dependence of the ensemble averages $\left\langle\Phi_{n}(t)\right\rangle(n=1,2,3,4)$ given by Eq. (26), for dipolar nonpolarizable molecules, at different values of the reorientation parameter $p=\mu E / k T$.

exponentially. The functions $\left\langle\Phi_{3}(t)\right\rangle$ grow steeply and, on traversing a maximum, decrease to a value of $\left\langle\Phi_{3}(\right.$ s.s. $\left.)\right\rangle$, whereas the $\left\langle\Phi_{4}(\mathrm{t})\right\rangle$, after passing through minimal short-time values, increase to $\left\langle\Phi_{\mathbf{4}}(\right.$ s.s. $\left.)\right\rangle$ by way of negative values.

The functions $\left\langle\varphi_{n}(t)\right\rangle$ describing the decay of two-dimensional rotational diffusion when the pulse $g(t)$ is switched off jumpwise at the moment of time $T_{0}>0$ is obtained on insertion of $g\left(\iota>T_{0}\right)=0$ into Eqs. (18). Then

$$
\left\langle\dot{\varphi}_{n}\right\rangle+\frac{1}{\tau_{n}}\left\langle\varphi_{n}\right\rangle=0
$$

and obviously the decay functions are

$$
\left\langle\varphi_{n}(t>T)\right\rangle=\left\langle\varphi_{n}\left(T_{0}\right)\right\rangle \mathrm{e}^{-t / \tau_{n}} .
$$

Each of the $n$-th two-dimensional rotation functions decreases exponentially in accordance with the $n$-th relaxation time.

\subsection{Another particular case: nondipolar $(p=0)$ anisotropically polarizable $(q \neq 0)$ molecules}

In the case of a statistical ensemble of nondipolar though anisotropically polarizable molecules, the functions $\left\langle\Phi_{n}(t)\right\rangle$ are mutually related as follows (see Eq. (20)): 


$$
\begin{aligned}
& \left\langle\Phi_{n}(t)\right\rangle=\left\langle\Phi_{n}\right\rangle_{0} \mathrm{e}^{-t / \tau_{n}} \\
& \quad+\frac{n q}{2 \tau_{1}} \int_{0}^{t} \mathrm{e}^{-(t-u) / \tau_{n}} g^{2}(u)\left[\left\langle\Phi_{n-2}(u)\right\rangle-\left\langle\Phi_{n+2}(u)\right\rangle\right] \mathrm{d} u .
\end{aligned}
$$

Obviously they fall into two mutually independent sets of recurrential equations, the one for even $n$ and the other for odd $n$. If, at $t=0$, the pulse is still absent, the condition (23) gives

$$
\left\langle\Phi_{1}(0)\right\rangle=\left\langle\Phi_{n}(0)\right\rangle=0 \quad \text { for } n \neq 0 .
$$

Thus for all odd values of $n$ we have

$$
\left\langle\Phi_{n}(t)\right\rangle=0, \quad n \text { odd! }
$$

whereas for even $n$ we get

$$
\begin{aligned}
& \left\langle\dot{\Phi}_{2}\right\rangle+\frac{1}{\tau_{2}}\left\langle\Phi_{2}\right\rangle+\frac{q g^{2}(l)}{\tau_{1}}\left(\left\langle\Phi_{4}\right\rangle-1\right)=0, \\
& \left\langle\dot{\Phi}_{4}\right\rangle+\frac{1}{\tau_{4}}\left\langle\Phi_{4}\right\rangle+\frac{2 q g^{2}(l)}{\tau_{1}}\left(\left\langle\Phi_{6}\right\rangle-\left\langle\Phi_{2}\right\rangle\right)=0, \\
& \left\langle\dot{\Phi}_{6}\right\rangle+\frac{1}{\tau_{6}}\left\langle\Phi_{6}\right\rangle+\frac{3 q g^{2}(l)}{\tau_{1}}\left(\left\langle\Phi_{8}\right\rangle-\left\langle\Phi_{4}\right\rangle\right)=0 .
\end{aligned}
$$

From (33) with the initial condition $\left\langle\Phi_{0}(0)\right\rangle=1$, within an accuracy to terms proportional to $q^{4}$, we obtain the following solutions:

$$
\begin{aligned}
& \left\langle\Phi_{2}(t)\right\rangle=\frac{1}{4} q\left(1-\mathrm{e}^{-t / \tau_{2}}\right) \\
& +\frac{1}{16} q^{3}\left(\frac{1}{8}-\frac{1}{9} \mathrm{e}^{-t / \tau_{2}}-\frac{1}{72} \mathrm{e}^{-t / \tau_{4}}-\frac{t}{6 \tau_{2}} \mathrm{e}^{-t / \tau_{2}}\right)+\mathrm{O}\left(q^{5}\right) \\
& \left\langle\Phi_{4}(t)\right\rangle=\frac{1}{8} q^{2}\left(\frac{1}{4}-\frac{1}{3} \mathrm{e}^{t / \tau_{2}}+\frac{1}{12} \mathrm{e}^{-t / \tau_{1}}\right)+\frac{1}{192} q^{4}\left(\frac{1}{8}+\frac{1}{72} \mathrm{e}^{-t / \tau_{2}}-\frac{241}{1800} \mathrm{e}^{-t / \tau_{4}}\right. \\
& \left.\quad-\frac{1}{200} \mathrm{e}^{-t / \tau_{6}}-\frac{t}{3 \tau_{2}} \mathrm{e}^{-t / \tau_{2}}-\frac{7 l}{120 \tau_{4}} \mathrm{e}^{-t / \tau_{4}}\right)+\mathrm{O}\left(q^{6}\right) \\
& \left\langle\Phi_{6}(t)\right\rangle=\frac{1}{128} q^{3}\left(\frac{1}{3}-\frac{1}{2} \mathrm{e}^{-t / \tau_{2}}+\frac{1}{5} \mathrm{e}^{-t / \tau_{4}}-\frac{1}{30} \mathrm{e}^{-t / \tau_{6}}\right)+\mathrm{O}\left(q^{5}\right)
\end{aligned}
$$

whence the "nondipolar" two-dimensional rotational diffusion functions are seen to rise from initial values equal to $\left\langle\Phi_{n}(0)\right\rangle=0$ to the steady state values (for $t \gg \tau_{1}$ ):

$$
\begin{aligned}
& \left\langle\Phi_{2}(\text { s.s. })\right\rangle=\frac{1}{4} q\left(1+\frac{1}{32} q^{2}\right)+\mathrm{O}\left(q^{5}\right), \\
& \left\langle\Phi_{4}(\text { s.s. })\right\rangle=\frac{1}{32} q^{2}\left(1+\frac{1}{48} q^{2}\right)+\mathrm{O}\left(q^{6}\right), \\
& \left.\left\langle\Phi_{6} \text { (s.s. }\right)\right\rangle=\frac{1}{384} q^{3}+\mathrm{O}\left(q^{5}\right),
\end{aligned}
$$

in accordance with the even relaxation times $\tau_{2}, \tau_{4}$ and $\tau_{6}$. Equations (34) involve exponential terms, as well as nonexponential terms in $t \exp \left(-t / \tau_{2}\right)$ and $t \exp \left(-t / \tau_{4}\right)$. The rise curves of the functions (34) are plotted in Fig. 2 for the reorientational parameter values $q=0.1,0.2,0.3,0.4$ and 0.5 . 

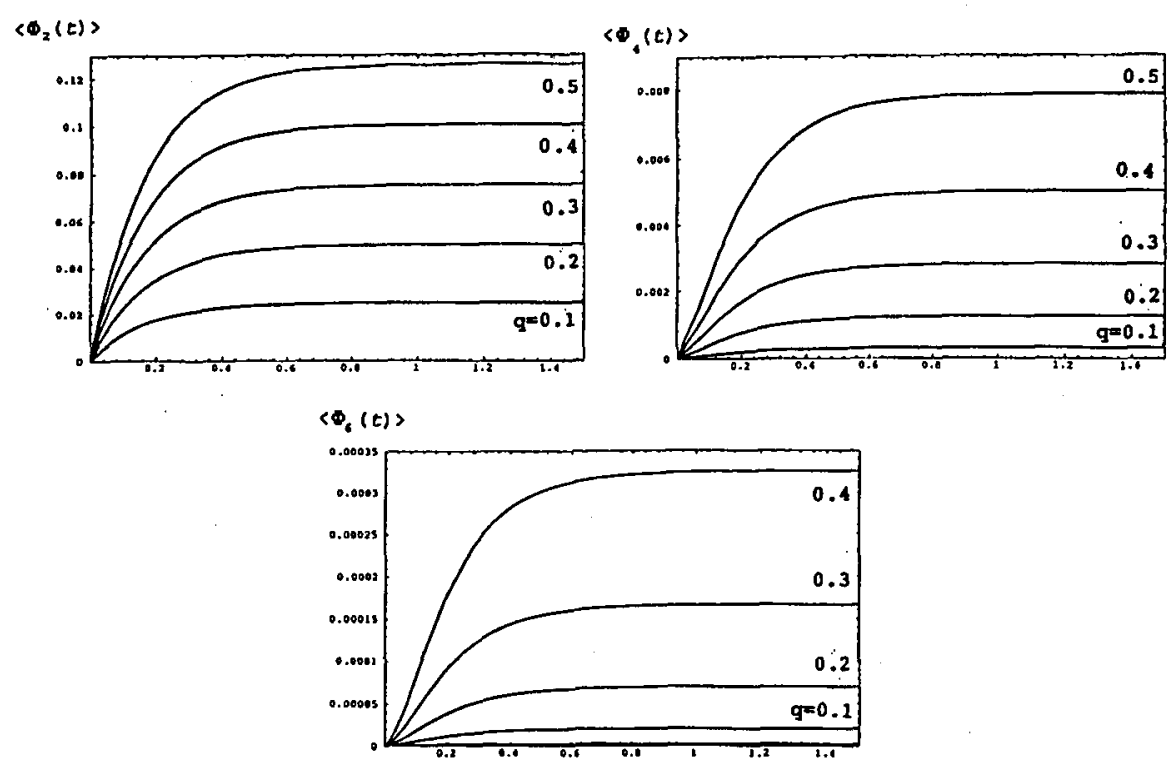

Fig. 2. Time dependence of the enscmble averages $\left\langle\Phi_{n}(t)\right\rangle(n=2,4,6)$ given by Eq. (34), for nondipolar anisotropically polarizable molecules, at different values of the reorientation parameter $q=\Delta \alpha E^{2} / 2 k T$.

Figure 2 shows that all the ensemble averages for nondipolar anisotropically polarizable molecules exhibit a qualitatively similar behaviour, though $\left\langle\Phi_{4}(t)\right\rangle$ and $\left\langle\Phi_{6}(t)\right\rangle$ initially deviate from exponentiality.

\section{Note added in proof}

Recently, Cisło and Dudek [13] have proposed a general method for the description of the diffusional time dependence of the ensemble averages $\left\langle\cos \left[\sum n_{k} \vartheta_{k}(\mathrm{t})\right]\right\rangle(k=1, \ldots, N)$ of $N$ rotators - interacting nonpolarizable dipolar molecules - subjected to a DC or cosine electric field. Applying the Fokker-Planck-Kramers equation, they obtained sets of recurrential equations for the ensemble averages for two rotators $\left\langle\cos \left[n_{1} v_{1}(t)+n_{2} \vartheta_{2}(\mathrm{t})\right]\right\rangle$ and for a single $\left\langle\Phi_{n}(\mathrm{t})\right\rangle=\langle\cos n \vartheta(t)\rangle$. The last set, Eq. (9) in Ref. [13], is identical with our (10) provided that the potential (2) is given by $V(\mathrm{t})=-V_{1}(\mathrm{t}) \cos v$.

Cisło and Dudek have solved the set analytically for the ensemble averages $\left\langle\Phi_{1}(\mathrm{t})\right\rangle$ with accuracy up to $\mathrm{O}\left(\mathrm{E}^{11}\right)$; their solution is in good agreement with their computer simulation, and the shape of their ensemble average corresponds to that of our $\left\langle\Phi_{1}(\mathrm{t})\right\rangle$ plot given by Eq. (26).

It should be noted that the two-dimensional Smoluchowski rotational diffusion equation has been used recently by Coffey et al. [14] to find exact analytic formulae for the correlation times for single domain ferromagnetic particles under the influence of an intcrnal uniaxial anisotropy potential and an external steady magnetic field [14]. 


\section{Acknowledgments}

The authors wish to thank Referce for all his remarks and for drawing attention to Ref. [13].

\section{References}

[1] W.T. Coffey, in: Dynamical Processes in Condensed Malter, Ed. M.W. Evans, Wiley, New York 1985, p. 69.

[2] H. Watanabe, A. Morita, Adv. Chem. Phys. 56, 255 (1984).

[3] S. Kielich, in: Dielectric and Related Molecular Processes, Vol. I, Ed. M. Davies, Chem. Soc., London 1972, p. 192.

[4] S. Kielich, Nonlinear Molecular Optics, Nauka, Moskva 1981 (in Russian).

[5] W.T. Coffey, J. Chem. Phys. 93, 724 (1990).

[6] W.T. Coffey, S.G. McGoldrick, Chem. Phys. 120, 1 (1988); W.T. Coffey, S.G. McGoldrick, K.P. Quinn, Chem. Phys. 125, 99 (1988).

[7] W.T. Coffey, Chem. Phys. 143, 171 (1990).

[8] J.L. Déjardin, J. Chem. Phys. 05, 576 (1991).

[9] J.L. Déjardin, G. Dcbiais, J. Chem. Phys. 95, 2787 (1991).

[10] A. Chełkowski, Dicleclric Physics, PWN, Elsevicr, Amstcrdam 1980.

[11] H. Benoit, Ann. Phys. 6, 561 (1951); J. Chim. Phys. 49, 517 (1952).

[12] Y.P. Kalmykov, K.P. Quinn, J. Chem. Phys. 05, 9142 (1991).

[13] J. Cislo, M.R. Dudek, J. Chem. Phys. 100, 1567 (1994).

[11] W.T. Coffey, D.S.F. Crothers, Yu.P. Kalınykov, E.S. Massawe, J.T. Waldron, J. Magn. Magn. Mater. 127, L254 (1993). 